\section{AB0256 PREDICTION OF DISEASE RELAPSES BY MULTIBIOMARKER DISEASE ACTIVITY SCORE AND AUTOANTIBODY STATUS IN RA PATIENTS TAPERING DMARD TREATMENT - AN UPDATE OF THE RETRO STUDY}

Melanie Hagen ${ }^{1}$, Koray Tascilar ${ }^{1}$, Michaela Reiser ${ }^{1}$, Larissa Valor ${ }^{1}$, Judith Haschka ${ }^{2}$, Arnd Kleyer ${ }^{1}$, Axel Hueber ${ }^{1}$, Bernhard Manger ${ }^{1}$, Georg Schett ${ }^{1}$, Jürgen Rech ${ }^{1}$, RETRO study group. ${ }^{1}$ Friedrich-Alexander-University ErlangenNuremberg (FAU), Department of Internal Medicine 3 - Rheumatology and Immunology, Universitätsklinikum Erlangen, Erlangen, Germany; ${ }^{2}$ St. Vincent Hospital, Medical University of Vienna, Vinforce study group, Vienna, Austria

Background: Achieving remission is the ultimate treatment goal in patients with rheumatoid arthritis (RA). With the development and wider use of highly effective disease modifying anti-rheumatic drugs (DMARD) about half of RA patients reach the disease remission state (1), raising the question about tapering or stopping anti-rheumatic treatment and appropriate predictors (2).

Objectives: The purpose was to analyze the effect of multi-biomarker disease activity (MBDA) score and anti-citrullinated protein (ACPA) on relapse rates in RA patients in sustained remission enrolled in the prospective randomized controlled RETRO study $(3,4,5)$.

Methods: MBDA scores and ACPA status were determined in the baseline samples of patients in sustained DAS28-ESR remission fulfilling RETRO inclusion criteria. Patients were unblended and either continued DMARDs (Control), tapered dose by $50 \%$ (Taper) or stopped DMARDs after tapering (Taper/Stop) for one year according to the RETRO study protocol. MBDA and ACPA status were used as relapse predictors. Relapse was defined as the loss of a DAS28-ESR remission. We calculated incidence of flares and 95\% Poisson confidence intervals by baseline ACPA and MBDA status in each study group (double negative, single positive, double positive). We compared the risk of flare in the treatment arms with a Cox regression model and calculated hazard ratios $(\mathrm{HR})$ and $95 \%$ confidence interval $(\mathrm{Cl})$ for relapses.

Results: Serum samples and follow-up data of 203 patients included in the RETRO trial were analyzed. A flare was observed in $8 / 59$ patients $(13.6 \%)$ in the Control group, 24/60 (40.0\%) patients in the Taper group and $37 / 68(54.4 \%)$ patients in the Taper/Stop group among the 187 patients that completed their 1-year follow-up. HR $(95 \% \mathrm{Cl})$ for a relapse was 3.43 (1.54-7.66) in the taper group and 5.32 (2.47-11.46) for the control group. HR of flare of a positive MBDA and ACPA was 4.00 (1.72-9.31) compared to a negative MBDA and ACPA. Flare incidence did not differ with baseline MBDA/ACPA status in the control group, whereas in the taper/stop group, number of positive biomarkers could identify three distinct subgroups with a graded incidence of flare (Figure).

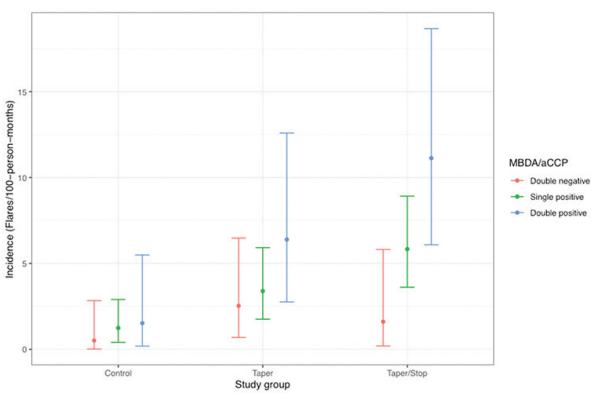

Conclusion: Tapering or stopping DMARDs after stable remission was associated with an increased risk of RA flares. Incidence of flares in ACPA/ MBDA double-negative patients after tapering and stopping RA treatment was comparable to those that continued treatment within the precision limits of our subgroups. Lack of blinding is a shortcoming of our study.

\section{REFERENCES}

[1] Aga AB et al. Ann Rheum Dis. 2013;74:381-8.

[2] Schett $G$ et al. Ann Rheum Dis. 2016 Aug;75(8):1428-373.

[3] Haschka J et al, Ann Rheum Dis. 2016;75:45-51.

[4] Rech J et al, Ann Rheum Dis. 2015; Oct 19.

[5] Hagen M et al, J Rheum. 2018

Acknowledgement: Following further colleagues participated in the RETRO trial: C.Figueiredo, J.Cobra, HP.Tony, S.Finzel, S.Kleinert, J.Wendler, F.Schuch, M.Ronneberger, M.Feuchtenberger, M.Fleck, K.Manger, W. Ochs, M.Schmitt-Haendle, HM.Lorenz, H.Nüsslein, R. Alten, J.Henes, K. Krüger
Disclosure of Interests: Melanie Hagen: None declared, Koray Tascilar: None declared, Michaela Reiser: None declared, Larissa Valor: None declared, Judith Haschka: None declared, Arnd Kleyer Grant/research support from: Lilly, Consultant for: Lilly, Speakers bureau: Abbvie, Axel Hueber: None declared, Bernhard Manger: None declared, Georg Schett: None declared, Jürgen Rech Grant/research support from: Bristol-Myers Squibb and Celgene (greater than $\$ 10,000$ ), Consultant for: Bristol-Myers Squibb, Celgene, Chugai, GlaxoSmithKline, Janssen, Eli Lilly, Novartis, Roche, Sanofi Aventis, and UCB (in total more than $\$ 10,000$ ), Speakers bureau: BristolMyers Squibb, Celgene, Chugai, GlaxoSmithKline, Janssen, Eli Lilly, Novartis, Roche, Sanofi Aventis, and UCB (in total more than $\$ 10,000$ ) DOI: 10.1136/annrheumdis-2019-eular.4333

\section{AB0257 FATIGUE LEVELS PREDICT OTHER PATIENT REPORTED OUTCOMES AND DISEASE ACTIVITY SCORES: RESULTS FROM A LONGITUDINAL STUDY OF RA PATIENTS INITIATING BDMARD THERAPY}

Hilde Berner Hammer ${ }^{1}$, Brigitte Michelsen ${ }^{2}$, Sella Aarrestad Provan ${ }^{1}$, Till Uhlig ${ }^{1}$, Tore K. Kvien ${ }^{1} .^{1}$ Diakonhjemmet Hospital, Dept. of Rheumatology, Oslo, Norway, ${ }^{2}$ Hospital of Southern Norway Trust, Division of Rheumatology, Department of Medicine, Kristiansand, Norway

Background: Fatigue is a sensation of weakness and lack of energy which is common in patients with rheumatoid arthritis (RA), contributing to reduced quality of life. However, there are few studies on the longitudinal influence of fatigue on patient reported outcome measures (PROMs).

Objectives: The present study explores the impact of fatigue on PROMs, clinical, laboratory and ultrasound (US) assessments.

Methods: A total of 208 patients with established RA (mean (SD) age 53 (13) years, disease duration 10 (9) years, $81 \%$ women, $79 \%$ antiCCP positive) were examined when initiating bDMARDs and assessed at baseline and after 1, 2, 3, 6 and 12 months, including fatigue $(0-10$, as part of the RAID score), PROMs (joint pain VAS, patient's global disease activity VAS (PGA), MHAQ, pain catastrophizing (PC), SF-36 Mental Health scale score (SF36MH)), clinical examinations (performed by a study nurse including examiner's global disease activity VAS (EGA), 28 tender and swollen joint counts (TJC, SJC)) and laboratory variables (ESR and CRP). US examinations (semi-quantitative scoring (0-3)) of grey scale (GS) and power Doppler (PD) were performed of 36 joints and 4 tendons by one rheumatologist $(\mathrm{HBH}$; Siemens Acuson Antares, excellence version, $5-13 \mathrm{MHz}$ probe). The clinical disease activity scores (CDAS) DAS28, CDAI and SDAl were calculated for all visits. Correlations were assessed by Spearman's rho. The predictive value of baseline fatigue on several dependent variables (PROMs, CDAS, clinical assessments) at all visits after baseline was explored by use of multiple linear regression analysis with adjustment for demographic values (age, sex and disease duration) and baseline inflammatory activity (CRP and sum score GS).

Results: Fatigue levels diminished during follow-up (baseline median (IQR) 5 (3-7), 12 months 2 (1-5)). Table 1 shows the high cross-sectional correlations between fatigue and PROMs/CDAS scores, low correlations with $\mathrm{EGA} / \mathrm{SJC} / \mathrm{CRP}$ and lack of correlations with US assessments. Baseline fatigue predicted PROMs and CDAS scores at all examinations (table 2). No/low associations were found between baseline fatigue and SJC/EGA/ sum score PD at follow-up.

Conclusion: Fatigue was highly correlated with PROMS and composite scores at all timepoints. The level of baseline fatigue predicted PROMs and composite scores at follow-up, but not objective inflammatory measures. Thus, the degree of fatigue should be taken into account when composite scores are used for evaluation of inflammatory activity in established RA patients.

\section{Table 1}

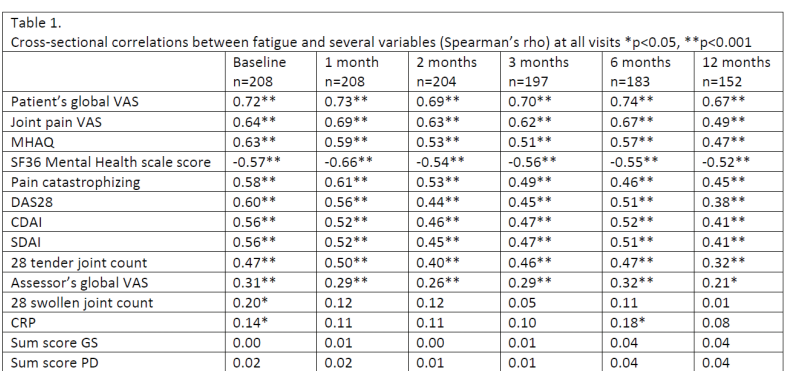

